\title{
Distinction of an Assortment of Deep Brain Stimulation Parameter Configurations for Treating Parkinson's Disease Using Machine Learning with Quantification of Tremor Response through a Conformal Wearable and Wireless Inertial Sensor
}

\author{
Robert LeMoyne', Timothy Mastroianni², Donald Whiting ${ }^{3}$, Nestor Tomycz ${ }^{4}$ \\ ${ }^{1}$ Department of Biological Sciences, Northern Arizona University, Flagstaff, AZ, USA \\ ${ }^{2}$ Cognition Engineering, Pittsburgh, PA, USA \\ ${ }^{3}$ Department of Neurosurgery, AHN Neuroscience Institute, Pittsburgh, PA, USA \\ ${ }^{4}$ AHN Department of Neurosurgery, Pittsburgh, PA, USA \\ Email: robert.lemoyne@nau.edu, rlemoyne07@gmail.com
}

How to cite this paper: LeMoyne, R., Mastroianni, T., Whiting, D. and Tomycz, N. (2020) Distinction of an Assortment of Deep Brain Stimulation Parameter Configurations for Treating Parkinson's Disease Using Machine Learning with Quantification of Tremor Response through a Conformal Wearable and Wireless Inertial Sensor. Advances in Parkinson's Disease, 9, 21-39.

https://doi.org/10.4236/apd.2020.93003

Received: July 7, 2020

Accepted: August 18, 2020

Published: August 21, 2020

Copyright $\odot 2020$ by author(s) and Scientific Research Publishing Inc. This work is licensed under the Creative Commons Attribution International License (CC BY 4.0).

http://creativecommons.org/licenses/by/4.0/

(c) (i) Open Access

\begin{abstract}
Deep brain stimulation offers an advanced means of treating Parkinson's disease in a patient specific context. However, a considerable challenge is the process of ascertaining an optimal parameter configuration. Imperative for the deep brain stimulation parameter optimization process is the quantification of response feedback. As a significant improvement to traditional ordinal scale techniques is the advent of wearable and wireless systems. Recently conformal wearable and wireless systems with a profile on the order of a bandage have been developed. Previous research endeavors have successfully differentiated between deep brain stimulation "On" and "Off" status through quantification using wearable and wireless inertial sensor systems. However, the opportunity exists to further evolve to an objectively quantified response to an assortment of parameter configurations, such as the variation of amplitude, for the deep brain stimulation system. Multiple deep brain stimulation amplitude settings are considered inclusive of "Off" status as a baseline, 1.0 $\mathrm{mA}, 2.5 \mathrm{~mA}$, and $4.0 \mathrm{~mA}$. The quantified response of this assortment of amplitude settings is acquired through a conformal wearable and wireless inertial sensor system and consolidated using Python software automation to a feature set amenable for machine learning. Five machine learning algorithms are evaluated: $\mathrm{J} 48$ decision tree, K-nearest neighbors, support vector machine,
\end{abstract}


logistic regression, and random forest. The performance of these machine learning algorithms is established based on the classification accuracy to distinguish between the deep brain stimulation amplitude settings and the time to develop the machine learning model. The support vector machine achieves the greatest classification accuracy, which is the primary performance parameter, and K-nearest neighbors achieves considerable classification accuracy with minimal time to develop the machine learning model.

\section{Keywords}

Parkinson's Disease, Deep Brain Stimulation, Wearable and Wireless Systems, Conformal Wearable, Machine Learning, Inertial Sensor, Accelerometer, Wireless Accelerometer, Hand Tremor, Cloud Computing, Network Centric Therapy, Python

\section{Introduction}

Deep brain stimulation offers a significant advance for the treatment of people with Parkinson's disease. With the considerable assortment of parameter configurations, such as amplitude, frequency, pulse width, and polarity, a patient specific treatment strategy is feasible [1] [2] [3]. The essence of efficacious intervention through the deep brain stimulation system is contingent upon converging the parameter configuration to an optimized setting, which can present a laborious process [2]-[7].

The deep brain stimulation system parameter optimization process relies upon the establishment of quantified feedback, such as through ordinal scales. However, the ordinal scale approach is inherently subjective, for which the reliability is controversial [3] [8] [9] [10] [11] [12]. A recommended alternative is the incorporation of a wearable and wireless inertial sensor system to provide feedback for intervention efficacy in a quantified and objective manner [3] [9] [13]-[28]. Recent technological evolutions have produced conformal wearable and wireless inertial sensor systems that impart minimal encumbrance relative to previous applications with a profile on the order of a bandage, such as the BioStamp nPoint [18] [29] [30].

The opportunities of the wearable and wireless system have been further evolved with the application of machine learning to ascertain considerable classification accuracy, such as for distinguishing between deep brain stimulation set to "On" and "Off". The machine learning classification endeavors have relied upon the operation of the Waikato Environment for Knowledge Analysis (WEKA) [3] [22] [23] [24] [25] [28] [29] [30]. WEKA presents an assortment of machine learning classification algorithms [31] [32] [33]. These machine learning algorithms offer contextually unique performance capabilities, and the most appropriate machine learning algorithm is integrally correlated with the intended application [24] [25] [28]. Beyond the scope of differentiating the "On" 
and "Off" status settings for deep brain stimulation exists the opportunity to distinguish between actual parameter configurations for deep brain stimulation through machine learning, such as with the variation of the amplitude parameter in conjunction with an assortment of machine learning algorithms.

The objective of the research endeavor is to evaluate the efficacy of machine learning algorithms with respect to the parametric variation of deep brain stimulation for the treatment of Parkinson's disease with the BioStamp nPoint providing quantified feedback. Five machine learning algorithms are considered: J48 decision tree, K-nearest neighbors, support vector machine, logistic regression, and random forest. The amplitude for deep brain stimulation is the selected parameter for variation respective of the following settings: "Off" status as a baseline, $1.0 \mathrm{~mA}, 2.5 \mathrm{~mA}$, and $4.0 \mathrm{~mA}$. Two machine learning performance parameters are considered for determining the most suitable algorithm: classification accuracy and time to develop the machine learning model.

\section{Background}

\subsection{Parkinson's Disease and Traditional Therapy}

With respect to the United States of America, approximately one million people have been diagnosed with Parkinson's disease, which is neurodegenerative and proportional to age [34] [35] [36]. The neurological basis for Parkinson's disease is associated with degeneration of the substantia nigra, which leads to diminished dopamine available for the caudate and putamen [34] [37]. Parkinson's disease characteristically involves the movement disorder of resting tremor, which has an approximate frequency of four to five per second [34] [38].

Traditional medication therapy for Parkinson's disease involves the prescription of levodopa [34] [37] [39]. Occasionally the medication therapy diminishes in efficacy, and an alternative intervention is sought, such as the thalamotomy and pallidotomy. These neurological techniques permanently disrupt pathways pertaining to the thalamus and globus pallidus internal segment [20] [34] [39] [40] [41] [42].

\subsection{Deep Brain Stimulation, an Advanced Concept for Treating Parkinson's Disease}

Dr. Alim-Louis Benabid developed deep brain stimulation as an advanced strategy for ameliorating Parkinson's disease symptoms during the later portion of the 1980's [1] [2] [3] [43] [44]. The primary subsystem of the deep brain stimulation system is the implantable pulse generator that is battery powered. The electric signal originating for the implantable pulse generator transmits through electrode leads that terminate at a prescribed deep brain neurological structure [4]. The ventral intermediate nucleus (VIM) and subthalamic nucleus (STN) are frequently utilized targets [2] [3] [5] [45].

Upon completion of the surgical procedure for the implementation of the deep brain stimulation system, the optimization of the parameter configuration is ascertained through the variation of the four available parameter settings: am- 
plitude, frequency, pulse width, and polarity [4] [6] [7]. The parameter configuration optimization process is inherently resource intensive while presenting a considerable challenge [2]-[7] [46]. Using current schemes, the acquisition of an optimal parameter configuration for deep brain stimulation can span multiple months [2] [5] [46]. Quantification of Parkinson's disease resting tremor can facilitate the determination of therapy efficacy [3] [8].

\subsection{Ordinal Scale Approach for Quantifying Parkinson's Disease Status}

The ordinal scale technique is a standard means for the determination of the Parkinson's disease status. An expert skilled clinician interprets the symptoms of a subject with Parkinson's disease in consideration of an established series of criteria. The Unified Parkinson's Disease Rating Scale is frequently utilized, and multiple other alternative ordinal scale are available [8] [9] [10] [11] [12]. However, a means for translating the perspectives of these multiple ordinal scales are not currently available. Furthermore, the reliability of the ordinal scale technique is a topic of controversy [8] [10]. Wearable and wireless systems with inertial sensors provide objective quantification of movement disorder features with a particular emphasis regarding Parkinson's disease [3] [9] [13]-[18] [20] [26] [27] [28] [47].

\subsection{Preliminary Wearable and Wireless Systems, such as the Smartphone, for Parkinson's Disease Quantification}

Functionally wearable inertial sensor systems equipped with accelerometers have been successfully applied for ascertaining therapy efficacy for movement disorders inclusive of Parkinson's disease [48]-[53]. The evolution to wearable and wireless inertial sensor systems has rendered tethering and manual techniques for uploading the signal data obsolete [3] [9] [13]-[18] [54] [55]. Locally wireless inertial sensor systems that are accessible to personal computers and functionally wearable have been successfully applied for the quantified characterization of symptomatic hand tremor for Parkinson's disease [20] [21] [56] [57].

LeMoyne and Mastroianni during 2010 succeeded in the preliminary demonstration of the smartphone as a wearable and wireless inertial sensor system for quantifying Parkinson's disease hand tremor through the smartphone's accelerometer. The acquired accelerometer signal data was conveyed wirelessly as an email attachment through the Internet for pending post-processing [19]. An immediate observed utility was that the experimental location and post-processing resources could be situated anywhere in the world with Internet access [3] [13]-[19].

\subsection{Integration of Wearable and Wireless Systems for Deep Brain Stimulation Treatment of Parkinson's Disease with Machine Learning Classification}

Wearable and wireless systems, such as the smartphone, have been successfully 
applied with machine learning to distinguish between various deep brain stimulation system settings for the treatment of movement disorders, such as Parkinson's disease and Essential tremor. The acquired inertial sensor signal data is consolidated into a feature set through post-processing. Considerable classification accuracy has been achieved for differentiating between "On" and "Off" settings for deep brain stimulation through the application of Waikato Environment for Knowledge Analysis (WEKA) [3] [22] [23] [24] [25] [28] [58]. WEKA offers an assortment of machine learning classification algorithms, such as the J48 decision tree, K-nearest neighbors, support vector machine, logistic regression, and random forest [31] [32] [33].

\subsection{Machine Learning for the Distinction of Deep Brain Stimulation Scenarios}

The combination of machine learning with wearable and wireless inertial sensor systems to provide quantified feedback for establishing a feature set differentiating deep brain stimulation system tuning scenarios has been advocated and successfully implemented with the Waikato Environment for Knowledge Analysis (WEKA) [3] [9] [13]-[30] [58]. WEKA consists of machine learning algorithms, such as the J48 decision tree, K-nearest neighbors, support vector machine, logistic regression, and random forest, which have disparate characteristic benefits regarding their application [29] [31] [32] [33] [58].

A general perspective of these machine learning algorithms is warranted. A major benefit for the J48 decision tree involves the ability to visualize the decision tree, which enables the research team to better comprehend the process for deriving the classification accuracy in consideration of the numeric attributes composing the feature set [31] [32] [33]. With respect to WEKA the J48 decision tree is derived from the C4.5 machine learning algorithm established by Quinlan, which is based on the application of information theory [31] [32] [33] [59] [60]. K-nearest neighbors incorporates an instance based algorithm with parameterized Euclidean distance. The origins of this machine learning technique date to the 1950's [31] [32] [33] [61]. The support vector machine originates from the 1990's by Cortes and Vapnik, and the algorithm establishes a hyperplane though the application of a kernel to the feature set with a support vector delineating the respective classes [31] [32] [33] [62] [63]. Logistic regression applies the ridge estimator for reducing the occurrence of overfitting while applying the sigmoid logit transform [31] [32] [33] [64]. Random forest constitutes a conceptual extension of the J48 decision tree algorithm, since a set of randomly established decision trees are applied for the improvement of performance [31] [32] [33] [65].

WEKA requires the development of an Attribute-Relation Format File (ARFF). The ARFF is comprised of the feature set, which involves a suitable group of numeric attributes that appropriately represent the respective classes [31] [32] [33]. Software automation for the post-processing of the inertial sensor 
signal data would facilitate the machine learning classification endeavor. The incorporation of software automation to consolidate inertial sensor signal data into an ARFF would benefit the determination of the efficacy of machine algorithms regarding the parametric variation of deep brain stimulation for the treatment of Parkinson's disease with the application of the conformally wearable and wireless BioStamp nPoint for providing quantified feedback.

\subsection{The BioStamp nPoint Conformal Wearable and Wireless Inertial Sensor for Quantifying Response to Deep Brain Stimulation for the Treatment of Parkinson's Disease}

The development of the BioStamp nPoint represents a significant evolution with respect to the domain of wearable and wireless inertial sensor systems. The BioStamp nPoint is highly flexible and conformal to effectively any aspect of the human anatomy. This conformal wearable can be secured through the application of an adhesive medium [18] [29] [30] [66]. This achievement of conformal wearable and wireless inertial sensor systems constitutes a significant advantage relative to previous devices, such as the larger and rigid smartphone, that require relatively more cumbersome adaptive mechanisms, such as elastic bands and gloves. Additionally, the BioStamp nPoint has a profile compatible to a bandage with a mass on the order of ten grams. The BioStamp nPoint has a mass on the scale of more than an order of magnitude less than the standard smartphone [3] [13]-[20] [22] [23] [24] [25] [27] [28] [29] [30] [58] [66].

The BioStamp nPoint is equipped with connectivity to a secure Cloud computing environment with wireless operation through the use of a smartphone and tablet. After the recording of a series of experimental inertial sensor data, the data is wirelessly transmitted to the secure Cloud computing environment for later post-processing, such as machine learning classification [18] [29] [30] [66]. Additionally, the BioStamp nPoint is certified as a $510(\mathrm{k})$ medical device by the FDA. This certification permits the acquisition of medical grade data [66].

The characteristics of the BioStamp nPoint underscore the unique utility of the device for the quantification of hand tremor symptoms for Parkinson's disease. The objective is to apply an assortment of machine learning algorithms for differentiating deep brain stimulation as a therapy intervention for Parkinson's disease respective of multiple parameter configuration settings. The selected parameter configuration is the amplitude $(1.0 \mathrm{~mA}, 2.5 \mathrm{~mA}$, and $4.0 \mathrm{~mA})$, and the "Off" status is included as a baseline. Five machine learning algorithms available with WEKA are selected: J48 decision tree, K-nearest neighbors, support vector machine, logistic regression, and random forest.

\section{Material and Methods}

One female subject with an age in the mid-60's diagnosed with Parkinson's disease in 2011 was selected for this preliminary demonstration from the perspective of engineering proof of concept. The subject has been receiving deep brain 
stimulation regarding the bilateral subthalamic nucleus. Informed consent was confirmed, and the research was conducted at Allegheny General Hospital in agreement with ethical clearance.

Since the BioStamp nPoint has a profile comparable to a bandage, the mounting of the device is applied to the dorsum of the hand through an adhesive medium with longitudinal alignment symmetrical to the third metacarpal. Similar mounting techniques have been successfully applied for quantifying Parkinson's disease through the use of wearable and wireless systems [19] [20] [21] [23] [24] [25] [29] [30]. Figure 1 presents the representative mounting technique, and Figure 2 displays the BioStamp nPoint and supporting apparatus.

Five machine learning classification algorithms available through WEKA have been designated for testing and evaluation: J48 decision tree, K-nearest neighbors, support vector machine, logistic regression, and random forest. There are two primary performance parameters considered with respect to the machine learning algorithms: classification accuracy and time to develop the machine learning model. Tenfold cross validation was incorporated [31] [32] [33].

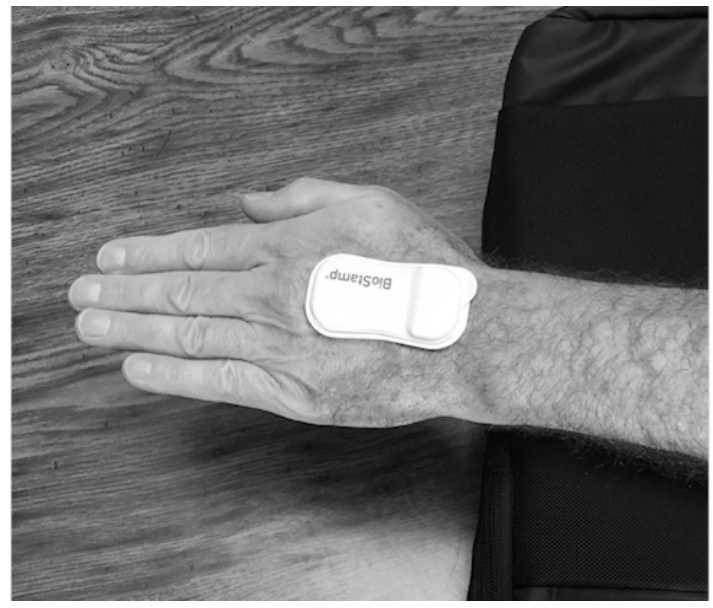

Figure 1. The BioStamp nPoint mounted by adhesive medium to the dorsum of the hand.

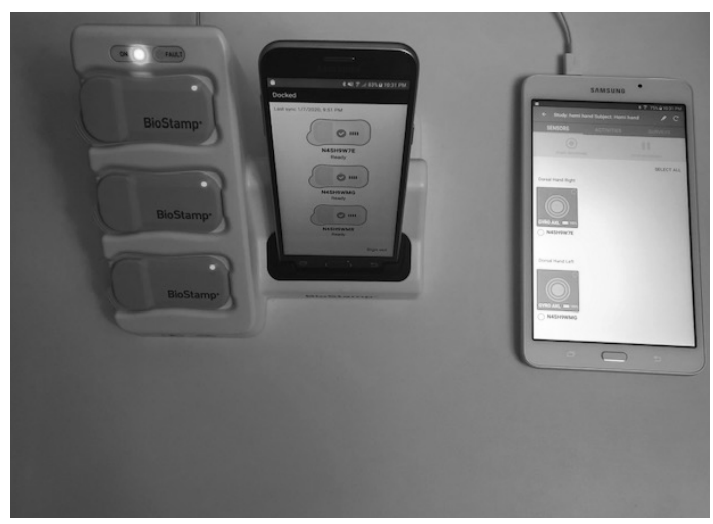

Figure 2. The BioStamp nPoint conformal wearable and wireless inertial sensor system and supporting apparatus, such as the smartphone, tablet, and docking station. 
The operation of WEKA requires the organization of an ARFF, which consists of a feature set with representative numeric attributes [31] [32] [33]. Software automation was applied to consolidate the acquired accelerometer magnitude signal data to a feature set in a syntactic manner suitable for the ARFF, and Python was selected as the programming language. The selected numeric attributes were a series of five descriptive statistics: maximum, minimum, mean, standard deviation, and coefficient of variation, which were established based on previous related machine learning classification research [22] [23] [24] [25] [28] [29] [30] [67].

The experiment involved the recording of accelerometer signal data from the BioStamp nPoint representing a conformal wearable and wireless system with parametric variation of the amplitude parameter for deep brain stimulation to the following settings: "Off" status as a baseline, 1.0 mA, 2.5 mA, and 4.0 mA. The BioStamp nPoint incorporated a sampling rate of $250 \mathrm{~Hz}$. The duration of the sampling for each deep brain stimulation amplitude setting was sufficient to acquire five trials lasting for two seconds. Contact with the table to the measured hand was prevented by extending the wrist of the subject beyond an elevated support.

The experimental protocol described below was applied for the four prescribed deep brain stimulation amplitude settings:

1) Mount the BioStamp nPoint by adhesive medium to the dorsum of the hand with a longitudinal and symmetric orientation respective of the third metacarpal.

2) Situate the respective forearm of the subject on an elevated support so that the Parkinson's disease hand tremor does not collide with the table.

3) Initiate the BioStamp nPoint recording with a duration that is capable of acquiring five trials for each prescribed deep brain stimulation amplitude parametric setting ("Off" status as a baseline, $1.0 \mathrm{~mA}, 2.5 \mathrm{~mA}$, and $4.0 \mathrm{~mA}$ ) for a two second duration.

4) With the completion of recording the trial data, wirelessly transmit the acquired inertial sensor data to the secure Cloud computing environment.

\section{Results and Discussion}

\subsection{Results}

The progressive increase of the amplitude parameter for deep brain stimulation induces the attenuation of Parkinson's disease hand tremor. The attenuating trend of Parkinson's disease hand tremor is quantified through the BioStamp nPoint, which constitutes a conformal wearable and wireless inertial sensor system, and the three dimensional orthogonal accelerometer signal is post-processed to the respective acceleration magnitude using Python. The deep brain stimulation system set to "Off" status represents a baseline for Parkinson's disease hand tremor as demonstrated by Figure 3. As the deep brain stimulation amplitude parameter is incrementally increased the acceleration magnitude of the Parkin- 
son's disease hand tremor is diminished as illustrated in Figure 4 with an amplitude of $1.0 \mathrm{~mA}$, Figure 5 with an amplitude of $2.5 \mathrm{~mA}$, and Figure 6 with an amplitude of $4.0 \mathrm{~mA}$.

Observation of Figures 3-6 infers perceptible distinction with regards to the deep brain stimulation set to "Off" status as a baseline, amplitude set to $1.0 \mathrm{~mA}$, amplitude set to $2.5 \mathrm{~mA}$, and amplitude set to $4.0 \mathrm{~mA}$. The acceleration magnitude is consolidated into a feature set consisting of five numeric attributes based on descriptive statistics: maximum, minimum, mean, standard deviation, and coefficient of variation. The derivation of the feature set is precedentially established from previous successful machine learning classification utilizing wearable and wireless inertial sensor systems [22] [23] [24] [25] [28] [29] [30] [67].

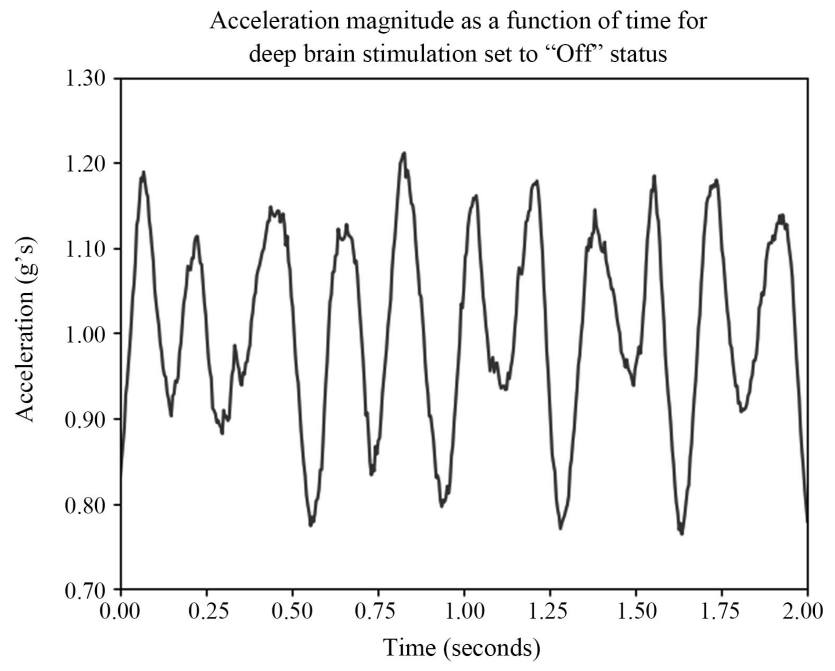

Figure 3. The acceleration magnitude of Parkinson's disease hand tremor quantified by the BioStamp nPoint conformal wearable and wireless inertial sensor system with deep brain stimulation set to "Off" status.

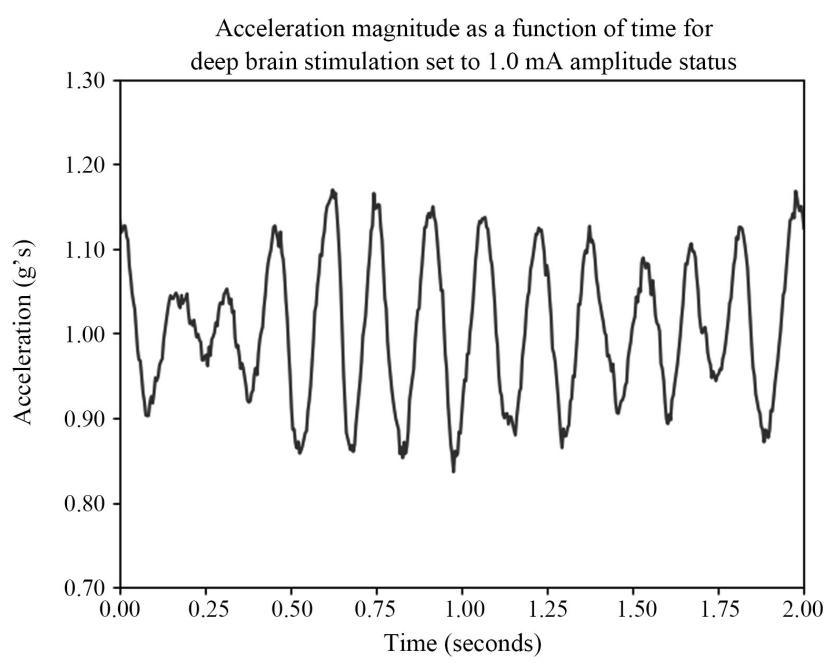

Figure 4. The acceleration magnitude of Parkinson's disease hand tremor quantified by the BioStamp nPoint conformal wearable and wireless inertial sensor system with deep brain stimulation amplitude set to $1.0 \mathrm{~mA}$. 


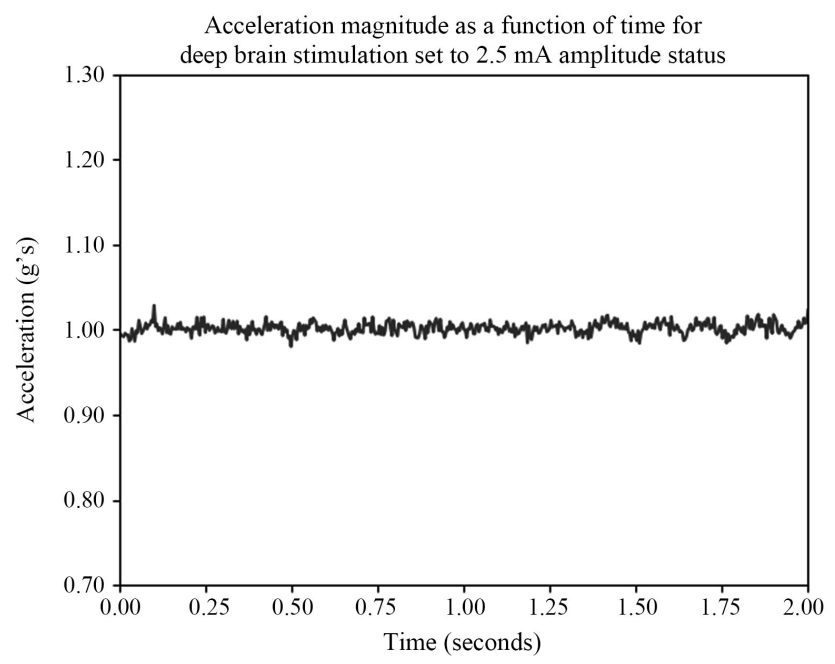

Figure 5. The acceleration magnitude of Parkinson's disease hand tremor quantified by the BioStamp nPoint conformal wearable and wireless inertial sensor system with deep brain stimulation amplitude set to $2.5 \mathrm{~mA}$.

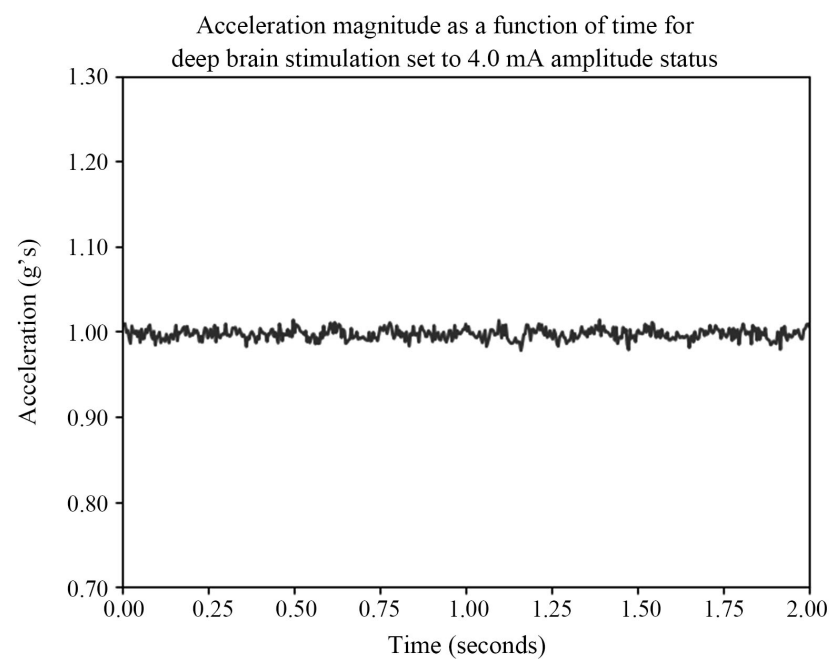

Figure 6. The acceleration magnitude of Parkinson's disease hand tremor quantified by the BioStamp nPoint conformal wearable and wireless inertial sensor system with deep brain stimulation amplitude set to $4.0 \mathrm{~mA}$.

Using the Waikato Environment for Knowledge Analysis (WEKA) five machine learning algorithms are selected for the ability to differentiate Parkinson's disease hand tremor quantified by the BioStamp nPoint conformal wearable and wireless inertial sensor system with respect to deep brain stimulation set to "Off" status as a baseline, amplitude set to $1.0 \mathrm{~mA}$, amplitude set to $2.5 \mathrm{~mA}$, and amplitude set to $4.0 \mathrm{~mA}$ :

- J48 decision tree

- K-nearest neighbors

- Support vector machine

- Logistic regression

- Random forest 
These machine learning algorithms are evaluated for the performance in terms of classification accuracy and time to derive the machine learning model. In association to classification accuracy the confusion matrix is addressed to further define the nature of misclassifications.

All five machine learning algorithms achieved considerable classification accuracy. Figure 7 presents their attained classification accuracy for differentiating between deep brain stimulation set to “Off” status as a baseline, amplitude set to 1.0 $\mathrm{mA}$, amplitude set to $2.5 \mathrm{~mA}$, and amplitude set to $4.0 \mathrm{~mA}$ for a subject with Parkinson's disease tremor. The Parkinson's disease tremor is quantified by a conformal wearable and wireless inertial sensor system. Figure 8 represents the time to develop the machine learning models that achieve their associated classification accuracy.

The J48 decision tree achieved 90\% classification accuracy. Two instances were misclassified. One instance involving deep brain stimulation set to "Off" status as a baseline was misclassified as being deep brain stimulation set to an amplitude of $1.0 \mathrm{~mA}$. The other instance involved deep brain stimulation set to an amplitude of $2.5 \mathrm{~mA}$ being misclassified to an amplitude of $1.0 \mathrm{~mA}$. The machine learning model was developed in less than 0.01 seconds.

K-nearest neighbors machine learning model was also developed within less than 0.01 seconds. K-nearest neighbors attained 95\% classification accuracy with one misclassified instance. One instance of deep brain stimulation set to "Off" status as a baseline was misclassified as an amplitude setting of $1.0 \mathrm{~mA}$.

The support vector achieved the greatest classification accuracy of $100 \%$. Although the time to develop the support vector machine learning model was relatively protracted. The time to establish the machine learning model was 0.19 seconds.

Logistic regression and random forest both achieve 95\% classification accuracy. They both misclassified an instance of deep brain stimulation set to "Off" status as a baseline as an amplitude setting of $1.0 \mathrm{~mA}$. The logistic regression required 0.07 seconds to develop, and the random forest machine learning model was developed in 0.09 seconds.

\subsection{Discussion}

The selection of the best machine learning algorithm is determined based on two performance parameters: classification accuracy and time to develop the machine learning model. In general, the classification accuracy is the primary performance parameter, and the time to derive the machine learning model is of secondary significance. Based on the organization of the performance parameters the support vector machine that attains $100 \%$ classification accuracy is the most appropriate machine learning algorithm for distinguishing deep brain stimulation amplitude parameter settings for a subject with Parkinson's disease tremor. Another observation is that the support vector machine requires the longest time to develop with a span of 0.19 seconds. 


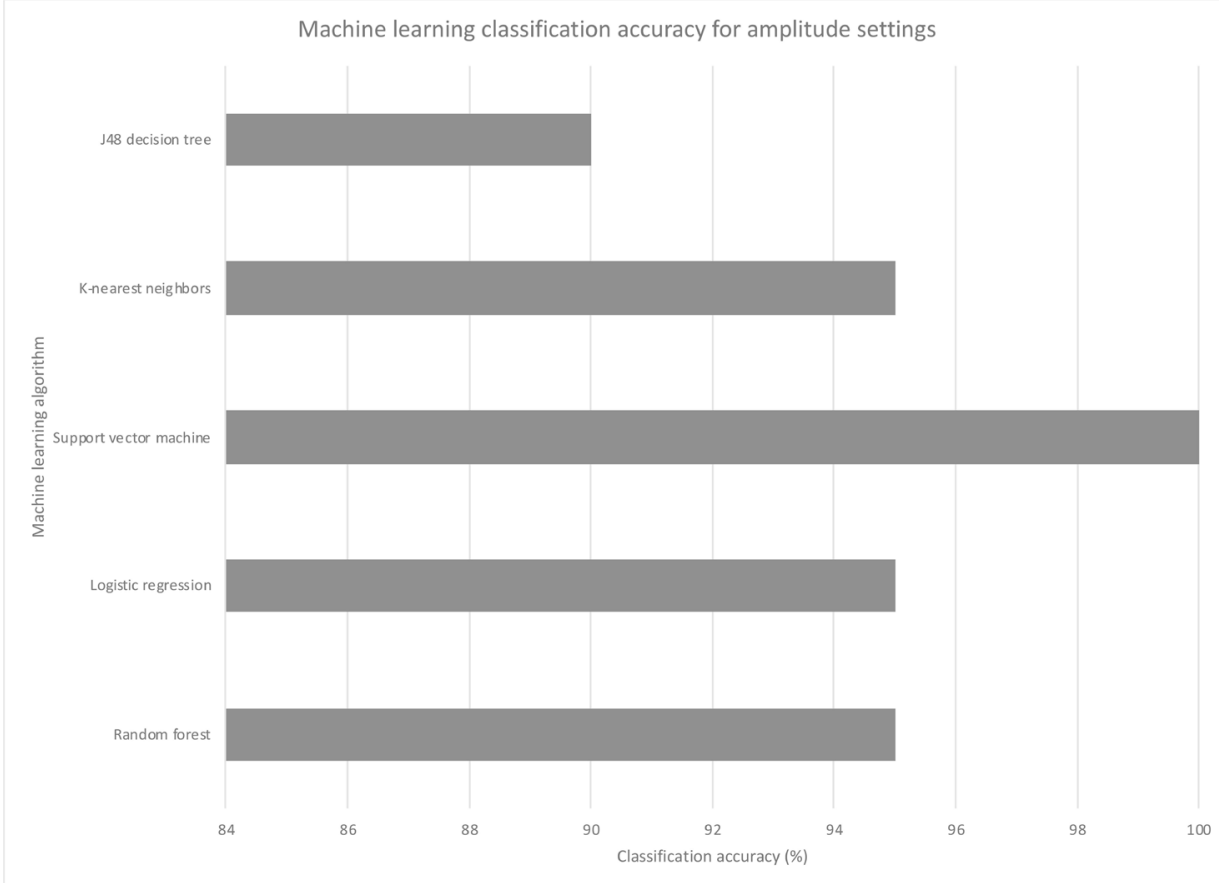

Figure 7. Machine learning classification accuracy achieved for the J48 decision tree, K-nearest neighbors, support vector machine, logistic regression, and random forest with respect to deep brain stimulation set to "Off" status as a baseline, amplitude set to $1.0 \mathrm{~mA}$, amplitude set to 2.5 $\mathrm{mA}$, and amplitude set to $4.0 \mathrm{~mA}$. The subject's Parkinson's disease hand tremor was quantified by a conformal wearable and wireless inertial sensor system.

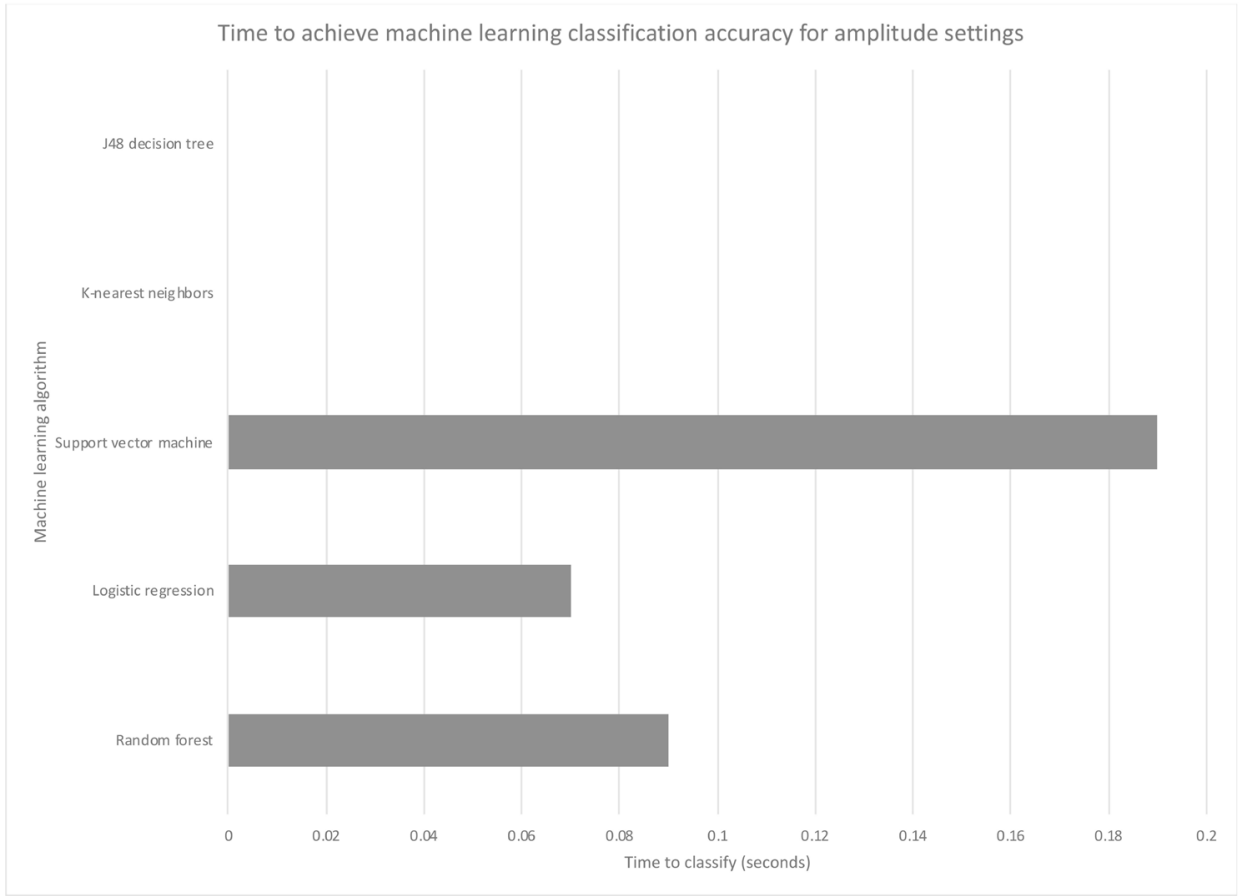

Figure 8. Time to develop the J48 decision tree, K-nearest neighbors, support vector machine, logistic regression, and random forest machine learning classification models. Note that the J48 decision tree and K-nearest neighbors machine learning algorithms require less than 0.01 seconds to develop their machine learning models. 
However, the time to develop the machine learning model may become more relevant in scenarios, for which the computational processing time is more protracted. This observation would be pertinent for a design requirement of deriving the classification accuracy through an associated wearable system rather than a Cloud computing environment. With respect to this design requirement scenario K-nearest neighbors would be most preferable, since the $95 \%$ classification accuracy is associated with a time to develop the machine learning classification model of less than 0.01 seconds.

These progressive evolutions further realize the broad objective of achieving real-time optimization of the parameter configuration for a deep brain stimulation system providing therapy for a person with a movement disorder. Additionally, the evaluation of more subjects is warranted in light of the successful preliminary research. These achievements further develop the presence of Network Centric Therapy, which synergizes the amalgamated capabilities of conformal wearable and wireless inertial sensor systems with data access to Cloud computing resources and acuity of machine learning to distinguish the quantified response to various therapy strategies. Network Centric Therapy has global healthcare implications as patients can be treated with internationally renowned medical talent from anywhere in the world [3] [17] [18] [26] [27] [28] [29] [30] [54] [68].

\section{Conclusions}

The efficacy of five machine learning algorithms (J48 decision tree, K-nearest neighbors, support vector machine, logistic regression, and random forest) has been successfully evaluated with respect to differentiating an assortment of deep brain stimulation parameter configurations, such as amplitude set to of "Off" status as a baseline, $1.0 \mathrm{~mA}, 2.5 \mathrm{~mA}$, and $4.0 \mathrm{~mA}$, for the treatment of Parkinson's disease. The composition of a feature set suitable for machine learning using WEKA is derived from the quantification of Parkinson's disease hand tremor based on a conformal wearable and wireless inertial sensor system with connectivity to a secure Cloud computing environment, which has a profile on the order of bandage and can be readily mounted about the dorsum of the hand by an adhesive medium. Post-processing of the recorded acceleration signal was facilitated through software automation enabled through Python.

In order to ascertain the best machine learning algorithm, two performance parameters were assigned. The primary performance parameter was classification accuracy, and secondary performance parameter was the time to develop the machine learning model. The support vector machine achieved $100 \%$ classification accuracy, but this machine learning algorithm required 0.19 seconds to construct the machine learning model, which is the greatest for the five machine learning algorithms under consideration. In the event that the time to construct the machine learning model becomes relatively more significant $\mathrm{K}$-nearest neighbors attains $95 \%$ classification accuracy with less than 0.01 seconds to develop the machine learning model. 
These progressive evolutions of conformal wearable and wireless inertial sensor systems with connectivity to Cloud computing resources in conjunction with machine learning further realize the development of Network Centric Therapy. In addition to the logistic implications of bridging the patient to clinician relation through the flexibility of Cloud computing resources through the expansive connectivity to the Internet exist the opportunities for treating movement disorders through data science strategies. The incremental evolutions establish the pathway for the development of real-time optimization of the parameter configuration for deep brain stimulation for the treatment of movement disorders, such as Parkinson's disease, in a closed-loop context through quantified feedback derived from conformal wearable and wireless inertial sensor systems.

\section{Acknowledgements}

Our research team would like to extend our appreciation to Allegheny General Hospital for enabling this opportunity to contribute to the amelioration of movement disorder symptoms.

\section{Conflicts of Interest}

The authors declare no conflicts of interest regarding the publication of this paper.

\section{References}

[1] Benabid, A.L., Pollak, P., Louveau, A., Henry, S. and De Rougemont, J. (1987) Combined (Thalamotomy and Stimulation) Stereotactic Surgery of the VIM Thalamic Nucleus for Bilateral Parkinson Disease. Applied Neurophysiology, 50, 344-346. https://doi.org/10.1159/000100803

[2] LeMoyne, R., Mastroianni, T., Whiting, D. and Tomycz, N. (2019) Chapter 4 Deep Brain Stimulation for the Treatment of Movement Disorder Regarding Parkinson's Disease and Essential Tremor with Device Characterization. In: Wearable and Wireless Systems for Healthcare II, Springer, Singapore, 37-51. https://doi.org/10.1007/978-981-13-5808-1_4

[3] LeMoyne, R., Mastroianni, T., Whiting, D. and Tomycz, N. (2019) Wearable and Wireless Systems for Healthcare II. Springer, Singapore. https://doi.org/10.1007/978-981-13-5808-1

[4] Amon, A. and Alesch, F. (2017) Systems for Deep Brain Stimulation: Review of Technical Features. Journal of Neural Transmission, 124, 1083-1091. https://doi.org/10.1007/s00702-017-1751-6

[5] LeMoyne, R., Mastroianni, T., Whiting, D. and Tomycz, N. (2019) Chapter 5 Surgical Procedure for Deep Brain Stimulation Implantation and Operative Phase with Postoperative Risks. In: Wearable and Wireless Systems for Healthcare II, Springer, Singapore, 53-63. https://doi.org/10.1007/978-981-13-5808-1_5

[6] Volkmann, J., Moro, E. and Pahwa, R. (2006) Basic Algorithms for the Programming of Deep Brain Stimulation in Parkinson's Disease. Movement Disorders, 21, S284-S289. https://doi.org/10.1002/mds.20961

[7] Isaias, I.U. and Tagliati, M. (2008) Chapter 20 Deep Brain Stimulation Programming for Movement Disorders. In: Tarsy, D., Vitek, J.L., Starr, P.A. and Okun, M.S., 
Eds., Deep Brain Stimulation in Neurological and Psychiatric Disorders, Springer, New York, 361-397. https://doi.org/10.1007/978-1-59745-360-8_20

[8] LeMoyne, R., Mastroianni, T., Whiting, D. and Tomycz, N. (2019) Chapter 3 Traditional Ordinal Strategies for Establishing the Severity and Status of Movement Disorders, such as Parkinson's Disease and Essential Tremor. In: Wearable and Wireless Systems for Healthcare II, Springer, Singapore, 25-36. https://doi.org/10.1007/978-981-13-5808-1_3

[9] LeMoyne, R., Coroian, C., Mastroianni, T., Opalinski, P., Cozza, M. and Grundfest, W. (2009) Chapter 10 the Merits of Artificial Proprioception, with Applications in Biofeedback Gait Rehabilitation Concepts and Movement Disorder Characterization. In: Barros De Mello, C.A., Ed., Biomedical Engineering, InTech, Vienna, Austria, 165-198. https://doi.org/10.5772/7883

[10] Ramaker, C., Marinus, J., Stiggelbout, A.M. and Van Hilten, B.J. (2002) Systematic Evaluation of Rating Scales for Impairment and Disability in Parkinson's Disease. Movement Disorders, 17, 867-876. https://doi.org/10.1002/mds.10248

[11] Goetz, C.G., Stebbins, G.T., Chmura, T.A., Fahn, S., Poewe, W. and Tanner, C.M. (2010) Teaching Program for the Movement Disorder Society-Sponsored Revision of the Unified Parkinson's Disease Rating Scale: (MDS-UPDRS). Movement Disorders, 25, 1190-1194. https://doi.org/10.1002/mds.23096

[12] Movement Disorder Society Task Force on Rating Scales for Parkinson's Disease (2003) The Unified Parkinson's Disease Rating Scale (UPDRS): Status and Recommendations. Movement Disorders, 18, 738-750. https://doi.org/10.1002/mds.10473

[13] LeMoyne, R. and Mastroianni, T. (2015) Chapter 23 Use of Smartphones and Portable Media Devices for Quantifying Human Movement Characteristics of Gait, Tendon Reflex Response, and Parkinson's Disease Hand Tremor. In: Rasooly, A. and Herold, K.E., Eds., Mobile Health Technologies: Methods and Protocols, Springer, New York, 335-358. https://doi.org/10.1007/978-1-4939-2172-0_23

[14] LeMoyne, R. and Mastroianni, T. (2017) Chapter 1 Smartphone and Portable Media Device: A Novel Pathway toward the Diagnostic Characterization of Human Movement. In: Mohamudally, N., Ed., Smartphones from an Applied Research Perspective, InTech, Rijeka, Croatia, 1-24. https://doi.org/10.5772/intechopen.69961

[15] LeMoyne, R. and Mastroianni, T. (2017) Chapter 6 Wearable and Wireless Gait Analysis Platforms: Smartphones and Portable Media Devices. In: Uttamchandani, D., Ed., Wireless MEMS Networks and Applications, Elsevier, New York, 129-152. https://doi.org/10.1016/B978-0-08-100449-4.00006-3

[16] LeMoyne, R. and Mastroianni, T. (2016) Telemedicine Perspectives for Wearable and Wireless Applications Serving the Domain of Neurorehabilitation and Movement Disorder Treatment. Telemedicine, SMGroup, Dover, DE, 1-10.

[17] LeMoyne, R. and Mastroianni, T. (2019) Chapter 7 Network Centric Therapy for Wearable and Wireless Systems. In: Dabove, P., Ed., Smartphones. Recent Innovations and Applications, Nova Science Publishers, Hauppauge, NY.

[18] LeMoyne, R. and Mastroianni, T. (2020) Chapter 2 Machine Learning Classification for Network Centric Therapy Utilizing the Multilayer Perceptron Neural Network. In: Vang-Mata, R., Ed., Multilayer Perceptrons: Theory and Applications, Nova Science Publishers, Hauppauge, NY, 39-76.

[19] LeMoyne, R., Mastroianni, T., Cozza, M., Coroian, C. and Grundfest, W. (2010) Implementation of an iPhone for Characterizing Parkinson's Disease Tremor through a Wireless Accelerometer Application. Proceedings of the 32nd Annual International Conference of the IEEE EMBS, Buenos Aires, 31 August-4 September 
2010, 4954-4958. https://doi.org/10.1109/IEMBS.2010.5627240

[20] LeMoyne, R. (2013) Wearable and Wireless Accelerometer Systems for Monitoring Parkinson's Disease Patients-A Perspective Review. Advances in Parkinson's Disease, 2, 113-115. https://doi.org/10.4236/apd.2013.24021

[21] LeMoyne, R., Mastroianni, T. and Grundfest, W. (2013) Wireless Accelerometer Configuration for Monitoring Parkinson's Disease Hand Tremor. Advances in Parkinson's Disease, 2, 62-67. https://doi.org/10.4236/apd.2013.22012

[22] LeMoyne, R., Tomycz, N., Mastroianni, T., McCandless, C., Cozza, M. and Peduto, D. (2015) Implementation of a Smartphone Wireless Accelerometer Platform for Establishing Deep Brain Stimulation Treatment Efficacy of Essential Tremor with Machine Learning. Proceedings of the 37 th Annual International Conference of the IEEE EMBS, Milan, 25-29 August 2015, 6772-6775.

https://doi.org/10.1109/EMBC.2015.7319948

[23] LeMoyne, R., Mastroianni, T., Tomycz, N., Whiting, D., Oh, M., McCandless, C., Currivan, C. and Peduto, D. (2017) Implementation of a Multilayer Perceptron Neural Network for Classifying Deep Brain Stimulation in "On” and "Off” Modes through a Smartphone Representing a Wearable and Wireless Sensor Application. Proceedings of the 47 th Annual Meeting of the Society for Neuroscience (Featured in Hot Topics, Top 1\% of Abstracts), Washington DC, 11-15 November 2017.

[24] LeMoyne, R., Mastroianni, T., McCandless, C., Currivan, C., Whiting, D. and Tomycz, N. (2018) Implementation of a Smartphone as a Wearable and Wireless Accelerometer and Gyroscope Platform for Ascertaining Deep Brain Stimulation Treatment Efficacy of Parkinson's Disease through Machine Learning Classification. Advances in Parkinson's Disease, 7, 19-30.

[25] LeMoyne, R., Mastroianni, T., McCandless, C., Currivan, C., Whiting, D. and Tomycz, N. (2018) Implementation of a Smartphone as a Wearable and Wireless Inertial Sensor Platform for Determining Efficacy of Deep Brain Stimulation for Parkinson's Disease Tremor through Machine Learning. Proceedings of the 48 th Annual Meeting of the Society for Neuroscience (Nanosymposium), San Diego, CA, 3-7 November 2018.

[26] LeMoyne, R., Mastroianni, T., Whiting, D. and Tomycz, N. (2019) Chapter 6 Preliminary Wearable and Locally Wireless Systems for Quantification of Parkinson's Disease and Essential Tremor Characteristics. In: Wearable and Wireless Systems for Healthcare II, Springer, Singapore, 65-78.

https://doi.org/10.1007/978-981-13-5808-1_6

[27] LeMoyne, R., Mastroianni, T., Whiting, D. and Tomycz, N. (2019) Chapter 7 Wearable and Wireless Systems with Internet Connectivity for Quantification of Parkinson's Disease and Essential Tremor Characteristics. In: Wearable and Wireless Systems for Healthcare II, Springer, Singapore, 79-97. https://doi.org/10.1007/978-981-13-5808-1_7

[28] LeMoyne, R., Mastroianni, T., Whiting, D. and Tomycz, N. (2019) Chapter 9 Assessment of Machine Learning Classification Strategies for the Differentiation of Deep Brain Stimulation "On” and "Off” Status for Parkinson's Disease Using a Smartphone as a Wearable and Wireless Inertial Sensor for Quantified Feedback. In: Wearable and Wireless Systems for Healthcare II, Springer, Singapore, 113-126. https://doi.org/10.1007/978-981-13-5808-1_9

[29] LeMoyne, R., Mastroianni, T., Whiting, D. and Tomycz, N. (2019) Network Centric Therapy for Deep Brain Stimulation Status Parametric Analysis with Machine Learning Classification. Proceedings of the 49th Annual Meeting of the Society for Neuroscience (Nanosymposium), Chicago, IL, 19-23 October 2019. 
[30] LeMoyne, R., Mastroianni, T., Whiting, D. and Tomycz, N. (2019) Preliminary Network Centric Therapy for Machine Learning Classification of Deep Brain Stimulation Status for the Treatment of Parkinson's Disease with a Conformal Wearable and Wireless Inertial Sensor. Advances in Parkinson's Disease, 8, 75-91. https://doi.org/10.4236/apd.2019.84007

[31] Hall, M., Frank, E., Holmes, G., Pfahringer, B., Reutemann, P. and Witten, I.H. (2009) The WEKA Data Mining Software: An Update. ACM SIGKDD Explorations Newsletter, 11, 10-18. https://doi.org/10.1145/1656274.1656278

[32] Witten, I.H., Frank, E. and Hall, M.A. (2011) Data Mining: Practical Machine Learning Tools and Techniques. Morgan Kaufmann, Burlington, MA.

[33] WEKA. http://www.cs.waikato.ac.nz/ ml/weka/

[34] Kandel, E.R., Schwartz, J.H. and Jessell, T.M. (2000) Chapter 43. Principles of Neural Science. McGraw-Hill, New York.

[35] Seeley, R.R., Stephens, T.D. and Tate, P. (2003) Chapter 14. Anatomy and Physiology. McGraw-Hill, Boston.

[36] LeMoyne, R., Mastroianni, T., Whiting, D. and Tomycz, N. (2019) Chapter 2 Movement Disorders: Parkinson's Disease and Essential Tremor-A General Perspective. In: Wearable and Wireless Systems for Healthcare II, Springer, Singapore, 17-24. https://doi.org/10.1007/978-981-13-5808-1_2

[37] Diamond, M.C., Scheibel, A.B. and Elson, L.M. (1985) Chapter 5. The Human Brain Coloring Book. Harper Perennial, New York.

[38] Bickley, L.S. and Szilagyi, P.G. (2003) Chapter 16. Bates' Guide to Physical Examination and History Taking. Lippincott Williams and Wilkins, Philadelphia.

[39] Nolte, J. and Sundsten, J.W. (2002) Chapter 19. The Human Brain: An Introduction to Its Functional Anatomy. Mosby, St. Louis, MO.

[40] Giller, C.A., Dewey, R.B., Ginsburg, M.I., Mendelsohn, D.B. and Berk, A.M. (1998) Stereotactic Pallidotomy and Thalamotomy Using Individual Variations of Anatomic Landmarks for Localization. Neurosurgery, 42, 56-65. https://doi.org/10.1097/00006123-199801000-00011

[41] Niranjan, A., Kondziolka, D., Baser, S., Heyman, R. and Lunsford, L.D. (2000) Functional Outcomes after Gamma Knife Thalamotomy for Essential Tremor and MS-Related Tremor. Neurology, 55, 443-446. https://doi.org/10.1212/WNL.55.3.443

[42] Young, R.F., Jacques, S., Mark, R., Kopyov, O., Copcutt, B., Posewitz, A. and Li, F. (2000) Gamma Knife Thalamotomy for Treatment of Tremor: Long-term Results. Journal of Neurosurgery, 93, 128-135. https://doi.org/10.3171/jns.2000.93.supplement_3.0128

[43] Williams, R. (2010) Alim-Louis Benabid: Stimulation and Serendipity. Lancet Neurology, 9, 1152. https://doi.org/10.1016/S1474-4422(10)70291-X

[44] Miocinovic, S., Somayajula, S., Chitnis, S. and Vitek, J.L. (2013) History, Applications, and Mechanisms of Deep Brain Stimulation. JAMA Neurology, 70, 163-171. https://doi.org/10.1001/2013.jamaneurol.45

[45] Yu, H. and Neimat, J.S. (2008) The Treatment of Movement Disorders by Deep Brain Stimulation. Neurotherapeutics, 5, 26-36. https://doi.org/10.1016/j.nurt.2007.10.072

[46] Pretto, T. (2007) Deep Brain Stimulation. Neurologist, 13, 103-104. https://doi.org/10.1097/01.nrl.0000258304.16124.e5

[47] LeMoyne, R., Coroian, C., Mastroianni, T. and Grundfest, W. (2008) Accelerometers for Quantification of Gait and Movement Disorders: A Perspective Review. 
Journal of Mechanics in Medicine and Biology, 8, 137-152.

https://doi.org/10.1142/S0219519408002656

[48] Schrag, A., Schelosky, L., Scholz, U. and Poewe, W. (1999) Reduction of Parkinsonian Signs in Patients with Parkinson's Disease by Dopaminergic versus Anticholinergic Single-Dose Challenges. Movement Disorders, 14, 252-255. https://doi.org/10.1002/1531-8257(199903)14:2\%3C252::AID-MDS1009\%3E3.0.CO; $2-\mathrm{N}$

[49] Keijsers, N.L.W., Horstink, M.W.I.M., Van Hilten, J.J., Hoff, J.I. and Gielen, C.C.A.M. (2000) Detection and Assessment of the Severity of Levodopa-Induced Dyskinesia in Patients with Parkinson's Disease by Neural Networks. Movement Disorders, 15, 1104-1111.

https://doi.org/10.1002/1531-8257(200011)15:6\%3C1104::AID-MDS1007\%3E3.0.C $\mathrm{O} ; 2-\mathrm{E}$

[50] Keijsers, N.L., Horstink, M.W. and Gielen, S.C. (2006) Ambulatory Motor Assessment in Parkinson's Disease. Movement Disorders, 21, 34-44. https://doi.org/10.1002/mds.20633

[51] Gurevich, T.Y., Shabtai, H., Korczyn, A.D., Simon, E.S. and Giladi, N. (2006) Effect of Rivastigmine on Tremor in Patients with Parkinson's Disease and Dementia. Movement Disorders, 21, 1663-1666. https://doi.org/10.1002/mds.20971

[52] Obwegeser, A., Uitti, R., Witte, R., Lucas, J., Turk, M. and Wharen, R. (2001) Quantitative and Qualitative Outcome Measures after Thalamic Deep Brain Stimulation to Treat Disabling Tremors. Neurosurgery, 48, 274-284. https://doi.org/10.1227/00006123-200102000-00004

[53] Kumru, H., Summerfield, C., Valldeoriola, F. and Valls-Solé, J. (2004) Effects of Subthalamic Nucleus Stimulation on Characteristics of EMG Activity Underlying Reaction Time in Parkinson's Disease. Movement Disorders, 19, 94-100. https://doi.org/10.1002/mds.10638

[54] LeMoyne, R. and Mastroianni, T. (2018) Wearable and Wireless Systems for Healthcare I. Springer, Singapore. https://doi.org/10.1007/978-981-10-5684-0

[55] Patel, S., Park, H., Bonato, P., Chan, L. and Rodgers, M. (2012) A Review of Wearable Sensors and Systems with Application in Rehabilitation. Journal of NeuroEngineering and Rehabilitation, 9, 1-17. https://doi.org/10.1186/1743-0003-9-21

[56] Giuffrida, J.P., Riley, D.E, Maddux, B.N. and Heldman, D.A. (2009) Clinically Deployable Kinesia Technology for Automated Tremor Assessment. Movement Disorders, 24, 723-730. https://doi.org/10.1002/mds.22445

[57] LeMoyne, R., Coroian, C. and Mastroianni, T. (2009) Quantification of Parkinson's Disease Characteristics Using Wireless Accelerometers. Proceedings of the International Conference on Complex Medical Engineering (CME-2009) of the IEEE/ICME, Tempe, AZ, 9-11 April 2009, 1-5. https://doi.org/10.1109/ICCME.2009.4906657

[58] LeMoyne, R., Mastroianni, T., McCandless, C., Whiting, D. and Tomycz, N. (2019) Evaluation of Machine Learning Algorithms for Classifying Deep Brain Stimulation Respective of "On" and "Off" Status. Proceedings of the 9th International IEEE/EMBS Conference on Neural Engineering (NER), San Francisco, CA, 20-23 March 2019, 483-488. https://doi.org/10.1109/NER.2019.8717095

[59] Quinlan, J.R. (1993) C4.5: Programs for Machine Learning. Morgan Kaufmann Publishers, San Mateo, CA.

[60] Quinlan, J.R. (1986) Induction of Decision Trees. Machine Learning, 1, 81-106. https://doi.org/10.1007/BF00116251

[61] Fix, E. and Hodges Jr., J.L. (1951) Discriminatory Analysis; Non-parametric Dis- 
crimination: Consistency Properties. USAF School of Aviation Medicine, Randolph Field, TX, Project Number: 21-49-004, Report Number: 4.

[62] Cortes, C. and Vapnik, V. (1995) Support Vector Networks. Machine Learning, 20, 273-297. https://doi.org/10.1007/BF00994018

[63] Vapnik, V.N. (1999) The Nature of Statistical Learning Theory. Springer-Verlag, New York. https://doi.org/10.1007/978-1-4757-3264-1

[64] Le Cessie, S. and Van Houwelingen, J.C. (1992) Ridge Estimators in Logistic Regression. Journal of the Royal Statistical Society. Series C (Applied Statistics), 41, 191-201. https://doi.org/10.2307/2347628

[65] Breiman, L. (2001) Random Forests. Machine Learning, 45, 5-32. https://doi.org/10.1023/A:1010933404324

[66] MC10 Inc. https://www.mc10inc.com/our-products\#biostamp-npoint

[67] LeMoyne, R., Heerinckx, F., Aranca, T., De Jager, R., Zesiewicz, T. and Saal, H.J. (2016) Wearable Body and Wireless Inertial Sensors for Machine Learning Classification of Gait for People with Friedreich's Ataxia. Proceedings of the 13 th International Conference on Wearable and Implantable Body Sensor Networks, San Francisco, CA, 14-17 June 2016, 147-151. https://doi.org/10.1109/BSN.2016.7516249

[68] LeMoyne, R., Mastroianni, T., Whiting, D. and Tomycz, N. (2019) Chapter 10 New Perspectives for Network Centric Therapy for the Treatment of Parkinson's Disease and Essential Tremor. In: Wearable and Wireless Systems for Healthcare II, Springer, Singapore, 127-128. https://doi.org/10.1007/978-981-13-5808-1_10 